\title{
Musik: Von der Koordination zur Kooperation
}

\section{Prosoziale Effekte von Musizieren, Singen und Tanzen}

M. Spitzer, UIm

Menschen sind die einzigen Primaten, die musizieren, singen und tanzen. Das tun sie nicht nur manchmal und (wie manche Tiere) zur Zeit der Balz (anders als die Tiere in geschlechtshormongeschwängerten schwach beleuchteten Clubs), sondern zu allen Zeiten und überall auf der Welt ( Abb. 1). Die Anthropologen, die dies herausgefunden haben, waren selber erstaunt darüber, dass es keine menschliche Kultur gibt, in der keine Musik vorkommt. ${ }^{1}$ Nun sind Menschen auch Gemeinschaftswesen, unter den Primaten die sozialsten, d. h. am stärksten auf Gemeinschaft ausgerichtet, und allein auf sich gestellt im Grunde nur sehr bedingt überlebensfähig. Könnte es da einen Zusammenhang geben?

Es gibt keine menschliche Kultur, in der keine Musik vorkommt.

Nun gibt es dazu viele Meinungen, die das bestätigen, aber auch andere, die daran zweifeln. Mein Freund Thomas - studierter Musiker mit viel Orchestererfahrung - beispielsweise sagte einmal, angesprochen auf Musik und Prosozialität (sinngemäß): „Wenn du wüsstest, wie wenig kollegial es in manchen Orchestern zugeht, würdest du dir nicht solche Gedanken machen." Wie

1 Ob es Kultur bei Tieren (Primaten und Nichtprimaten) gibt, wird diskutiert. Wenn ja, dann sind die - jeweils von älteren Artgenossen gelernten - Gesänge der Singvögel und Buckelwale herausragende Beispiele dafür (neben dem Werkzeuggebrauch von Krähen, die übrigens auch singen, und manchen nicht menschlichen Primaten; 5, 13).

Nervenheilkunde 2018; 37: 433-438

Korrespondenzadresse

Prof. Dr. Dr. Manfred Spitzer, Universitätsklinikum Ulm Klinik für Psychiatrie und Psychotherapie III

Leimgrubenweg 12, 89075 Ulm gut, dass es seit geraumer Zeit empirische Untersuchungen zu den prosozialen Effekten von Musik und Tanz gibt, die zum Teil erstaunliche Ergebnisse hervorbrachten.

Schon vor 30 Jahren publizierten die beiden Israelischen Psychologen Anat Anshel und David Kipper (1) eine Studie an 96 israelischen Männern im Alter von 22-41 Jahren (Mittelwert: 32), die in vier Gruppen $\mathrm{zu}$ jeweils 24 Personen randomisiert aufgeteilt wurden. In einem 2x2-Design mit den Faktoren Musik (ja/nein) und Aktivität (aktiv/passiv) wurden die vier Gruppen einer der folgenden Bedingungen zugeteilt:

- gemeinsam Singen (Musik/aktiv),

- gemeinsam Musik hören (Musik/passiv),
- gemeinsam einen Text vorlesen (keine Musik/aktiv),

- gemeinsam einen Film anschauen (keine Musik (passiv).

Das Ausmaß an Vertrauen in andere Personen wurde mittels eines Fragebogens ermittelt (18 Items aus dem insgesamt 27 Items umfassenden Griffin-Trust-Differential Questionaire). Die Bereitschaft zur Kooperation wurde mittels des Gefangenendilemma-Spiels erfasst, das jeweils in Dyaden gespielt wurde. Die Ergebnisse der Studie sind in Abbildung 2 zu sehen: Musik hat sowohl in der Form des aktiven Singens als auch des passiven Hörens einen signifikanten positiven Effekt auf das Vertrauen.

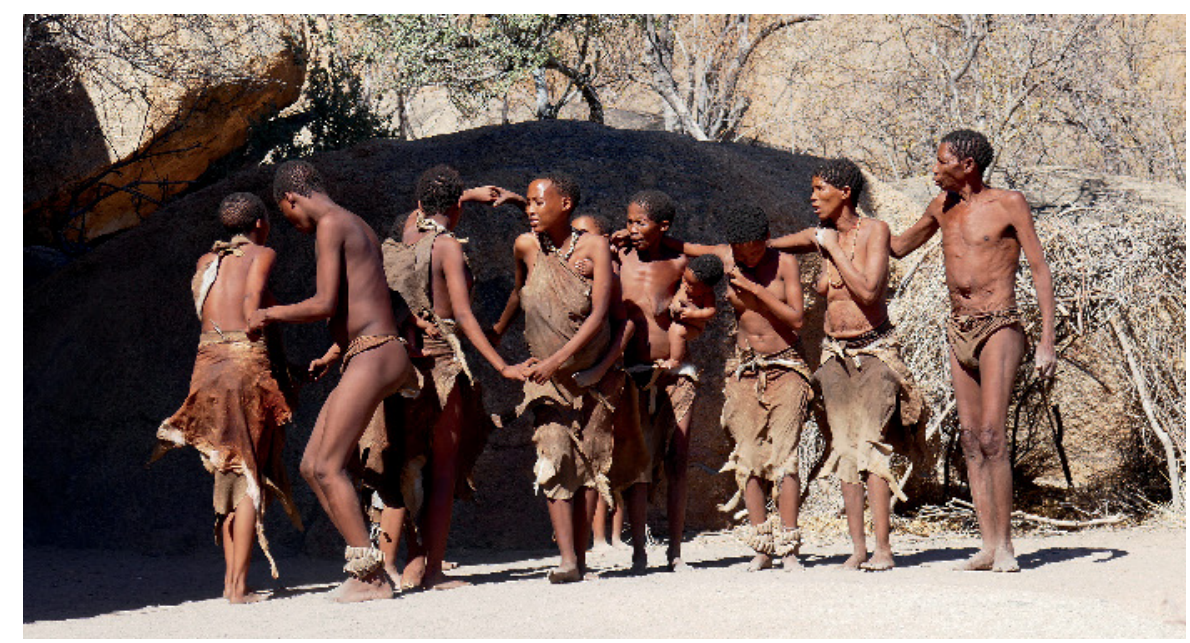

Abb. 1 Das gemeinsame Tanzen ist Bestandteil des Alltags bei den San in Namibia, die in nordöstlichen Landesteilen noch heute als Jäger und Sammler - fast - so leben wie vor Jahrtausenden in der Steinzeit. Ausnahmen sind die Gegenstände, die für den Kontakt mit Menschen aus der westlichen Zivilisation erforderlich sind, wie beispielsweise Geld aus dem Verkauf selbst hergestellter Schmuckwaren. Auch bei deren Herstellung aus kleinen runden Stücken der Schalen von Straußeneiern wie vor 75000 Jahren verwenden sie heute zum Bohren des Lochs zum Auffädeln einen Stahlbohrer. Auf meine Nachfrage meinte eine junge Frau mit dem Bohrer in der Hand während der Arbeit: "Stein geht schneller kaputt" . (Die Aufnahme entstand im "The Living Museum", in dem wechselnde Gruppen aus dem Nordosten in einer für Touristen erschlossenen Region gleichsam sich selber und ihre Lebensweise ausstellen. Sie können davon ihre von Steinzeitbedingungen kaum unterschiedene Lebensweise mit finanzieren, "gewöhnen" sich jedoch wegen der Rotation für jeweils nur wenige Wochen ins Museum nicht allzu sehr an die westliche Zivilisation.) 
Einundzwanzig Jahre später publizierten Scott Wiltermuth und Chip Heath von der Stanford University drei clever ausgedachte Experimente mit ähnlicher Fragestellung (16). Es ging den Autoren nicht nur um die Frage, ob es prosoziale Effekte von gemeinsamem Singen gibt, sondern auch um darum, ob das gemeinsame Bewegen - also Tanzen - einen ähnlichen Effekt hat.

Musik hat sowohl in der Form des aktiven Singens als auch des passiven Hörens einen signifikanten positiven Effekt auf das Vertrauen.

Im ersten an 30 Studenten (Durchschnittsalter 20 Jahre; 18 weiblich) durchgeführten Experiment machte der Versuchsleiter mit Dreiergruppen einen Spaziergang über den Campus der Uni, wobei er die Studenten entweder dazu anhielt, im Gleichschritt zu laufen oder nicht. Danach mussten sie ei- nen Fragebogen ausfüllen und dann war der Spaziergang beendet. Das Experiment aber nicht. Denn als nächstes wurde die Gruppe von einem zweiten Versuchsleiter übernommen, der mit der Gruppe nun für die Probanden völlig unabhängig vom Spaziergang - einen Test machte, der die soziale Einstellung jedes der drei Gruppenmitgliedern zu den anderen beiden erfassen sollte.

Das verwendete Weak-Link Game geht auf Ökonomen zurück, die bestimmte Entscheidungssituationen modellieren wollten, bei denen es immer darum geht, dass die Effektivität der Gesamtgruppe von der Effektivität des schwächsten Mitglieds abhängt. „Jede Kette ist nur so stark wie ihr schwächstes Glied“. Das Sprichwort ist nicht umsonst so bekannt, denn Beispiele für solche Situationen gibt es viele: Ein Geheimnis für sich behalten; erst mit dem Essen beginnen, wenn der Letzte da ist; oder ein Kapitel für ein Buch verfassen, das erst

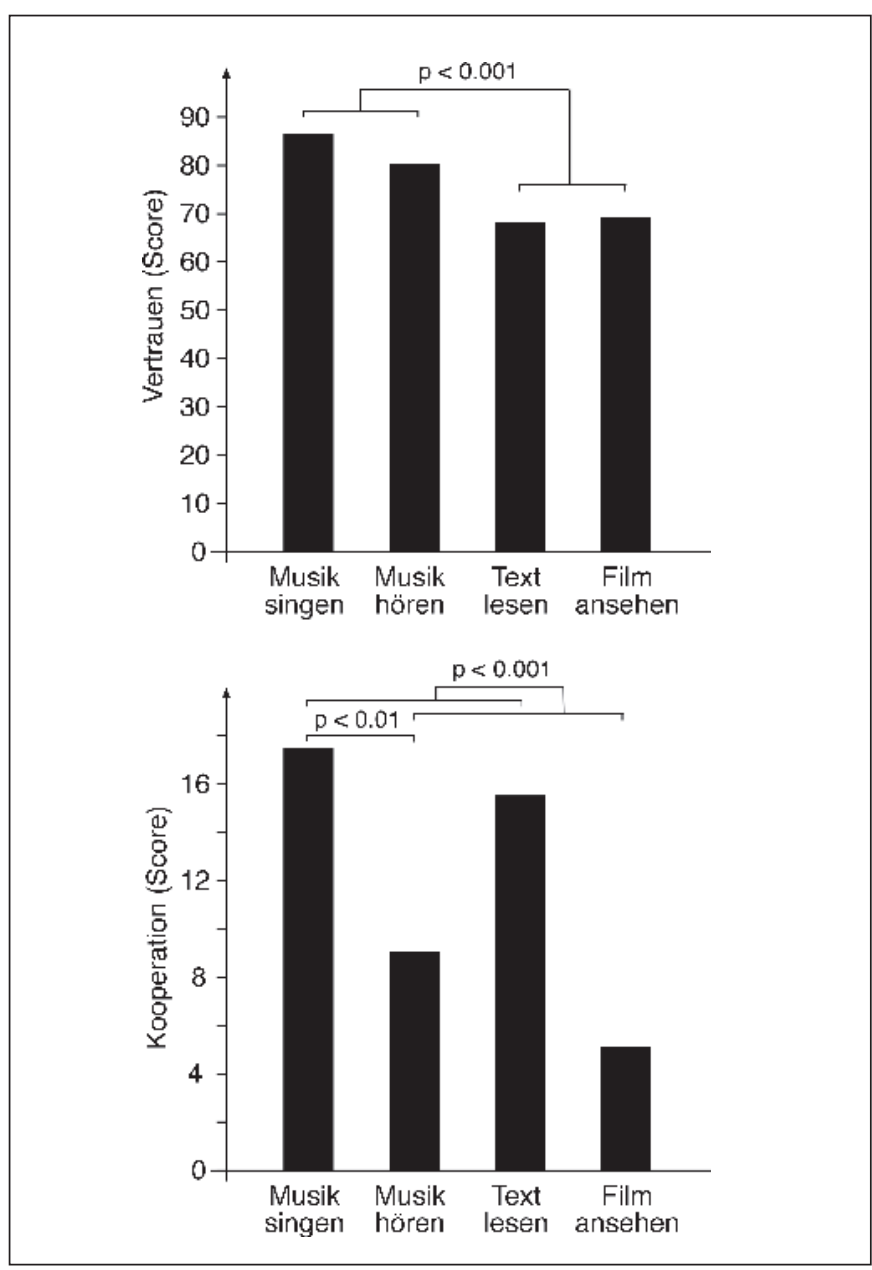

Abb. 2

Ergebnis der Studie von Anshel und Kipper (1). Oben: Musik (aktiv singen als auch passiv hören) hatte einen signifikanten positiven Effekt auf das Vertrauen (Haupteffekt für Musik in der Varianzanalyse: $p<0,001)$. Unten: Im Hinblick auf die Kooperation im Gefangenendilemma gab es in der Varianzanalyse einen Haupteffekt des aktiven Handelns (sowohl in Gestalt des Singens als auch des Vorlesens von Text; $p<0,001$ ); der Haupteffekt des Faktors "Musik" war mit $p=0,078$ nur als Trend vorhanden. Betrachtete man bei der Kooperation nur die beiden Musik-Gruppen, so war die Kooperation nach dem Singen signifikant größer $(p<0,01)$ als nach dem Musikhören.

gedruckt werden kann, wenn alle Kapitel geschrieben sind (15).

Jedes Mitglied der Dreiergruppe musste eine Zahl von 1 bis 7 aufschreiben. Danach bekommt jeder einen Geldbetrag ausbezahlt, dessen Höhe abhängt,

- von der Höhe der von ihm gewählten Zahl (je höher, desto mehr) und

- von der kleinsten durch einen der drei Spieler gewählten Zahl (je größer der Unterschied der eigenen Zahl zur kleinsten Zahl, desto weniger).

Was genau bei welcher Kombination von selbst gewählter Zahl und kleinster von den drei Spielern gewählter Zahl dem Spieler jeweils ausgezahlt wird, zeigt $>$ Tabelle 1 . Absprachen zwischen den Teilnehmern sind nicht möglich, denn sonst wäre die Sache einfach: Jeder wählt die 7 und bekommt den Höchstbetrag. Und weil man sich nicht absprechen kann, könnte es sich lohnen, nicht die $7 \mathrm{zu}$ wählen, denn dann bekommt man mehr ausgezahlt, wenn ein anderer auch nicht die 7 wählt. Das wiederum weiß jeder. Was der Test also letztlich misst, ist das Ausmaß des Vertrauens, das jeder der drei Spieler in die jeweils beiden anderen hat. Wenn ich die 1 wähle, bekomme ich auf jeden Fall 4,20 US Dollar. Wenn ich die 7 wähle, bekomme ich schlimmstenfalls (nämlich dann, wenn ein anderer die 1 wählt) nur 60 Cent.

Man spielte insgesamt sechs Runden. Jeder schrieb seine Zahl auf einen Zettel, der Versuchsleiter gab die kleinste gewählte Zahl an (wodurch jeder wusste, wie er selbst abgeschnitten hat, aber nicht wusste, wer von den anderen diese kleinste Zahl gewählt hat), und danach wurde die nächste Runde gespielt. Am Ende wurde eine der Runden per Zufall ausgewählt und die Auszahlung wurde gemäß dem Ergebnis dieser ausgewählten Runde durchgeführt. Dann sollten die Teilnehmer jeder für sich noch drei Fragen beantworten - auf einer Skala von 1 (gar nicht) bis 7 (sehr) -, die sich darauf bezogen,

- wie zusammengehörig sie sich beim Spaziergang mit den anderen beiden Teilnehmern gefühlt hätten,

- in wieweit sie den anderen beim Experiment vertraut hatten und

- wie glücklich sie sich jetzt gerade fühlten. $^{2}$ 
Tab. 1 Auszahlungsmatrix des Spiels „Schwächstes Kettenglied“ (Weak-Link Game; Werte aus 16). Die Höhe der Auszahlung an den einzelnen Spieler steigt mit seiner gewählten Zahl und fällt mit dem Abstand seiner gewählten Zahl zur kleinesten von allen gewählten Zahl.

\begin{tabular}{|c|c|c|c|c|c|c|c|c|}
\hline & & inster gewählt & Wert (von aller & rei Spielern) & & & & \\
\hline \multirow{8}{*}{$\begin{array}{l}\text { Selbst } \\
\text { gewähl- } \\
\text { ter Wert }\end{array}$} & & 7 & 6 & 5 & 4 & 3 & 2 & 1 \\
\hline & 7 & 7,80 US Dollar & 6,60 US Dollar & 5,40 US Dollar & 4,20 US Dollar & 3.00 US Dollar & 1,80 US Dollar & 0,60 US Dollar \\
\hline & 6 & & 7,20 US Dollar & 6,00 US Dollar & 4,80 US Dollar & 3,60 US Dollar & 2,40 US Dollar & 1,20 US Dollar \\
\hline & 5 & & & 6,60 US Dollar & 5,40 US Dollar & 4,20 US Dollar & 3.00 US Dollar & 1,80 US Dollar \\
\hline & 4 & & & & 6,00 US Dollar & 4,80 US Dollar & 3,60 US Dollar & 2,40 US Dollar \\
\hline & 3 & & & & & 5,40 US Dollar & 4,20 US Dollar & 3.00 US Dollar \\
\hline & 2 & & & & & & 4,80 US Dollar & 3,60 US Dollar \\
\hline & 1 & & & & & & & 4,20 US Dollar \\
\hline
\end{tabular}

Das Ergebnis des Experiments war eindeutig ( Abb. 3): Wer miteinander im Gleichschritt gelaufen war, fühlte sich stärker zusammengehörig, vertraute den anderen mehr und schrieb daher im Mittel höhere Zahlen auf als beim bloßen Spaziergang „ohne Tritt“ (wie man das „Nicht-imGleichschritt-Laufen“ im militärischen Kontext nennt). Im Hinblick auf Glücksgefühle gab es hingegen keinen Unterschied zwischen den Bedingungen „synchron laufen“ und „nicht synchron laufen“.

Im zweiten Experiment an 96 Teilnehmern (56\% weiblich, Durchschnittsalter 21 Jahre) ging es ebenfalls um synchrones Agieren, das wie folgt implementiert wurde: In Dreiergruppen hörten die Teilnehmer über Kopfhörer das gleiche Lied („O Kanada“; alle Studenten kamen aus den USA) und hielten alle einen Plastikbecher in der Hand. Per Zufall wurden die Dreiergruppen einer von vier Experimentalbedingungen zugeteilt. In der einfachsten Bedingung (Kontrolle) hörten die Teilnehmer das Lied, hielten den Plastikbecher (ohne Bewegung) in der Hand und lasen still für sich den Liedtext während sie das Lied hörten. In der zweiten Bedingung (synchron singen) sangen die Teilnehmer das Lied gleichzeitig, in der dritten sangen sie das Lied und bewegten zugleich den Plastikbecher im Rhythmus hin und her (Bedingung synchron singen und agieren) und in der vierten Bedingung (asynchron) hörten und

2 „How connected did you feel with the other participants during the walk?" „How much did you trust the other participants going into the exercise? „How happy do you feel?"“ $(1=$ not at all, $7=$ very much).

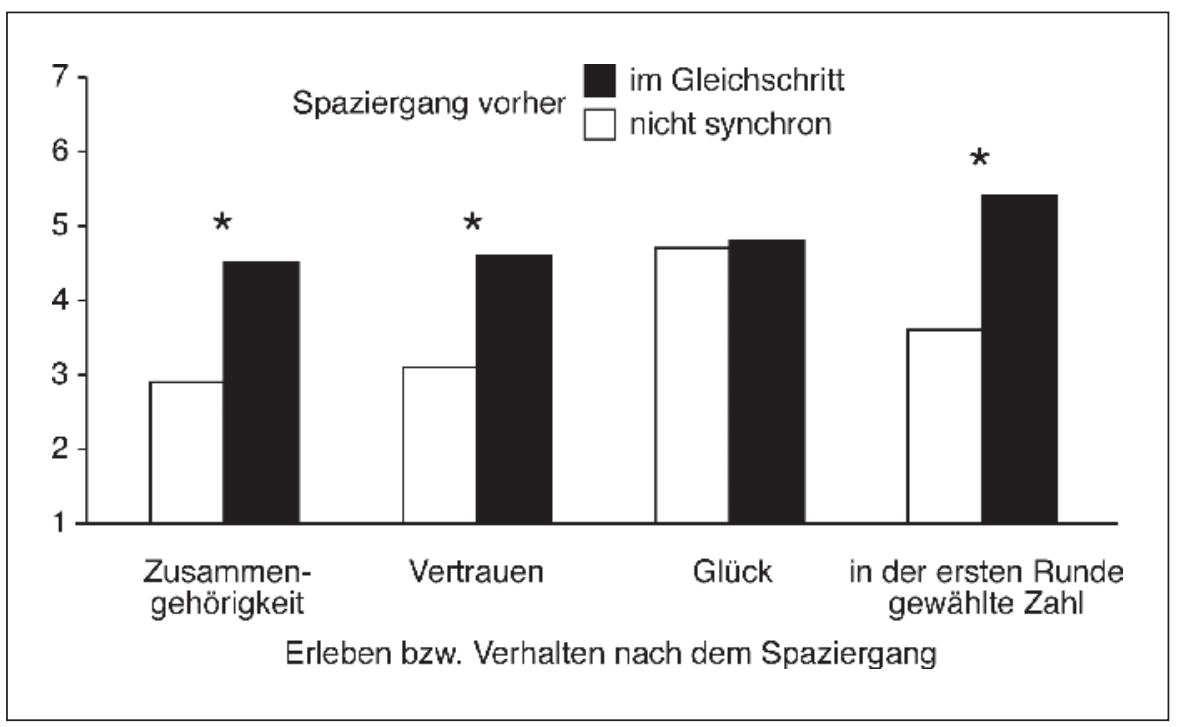

Abb. 3 Effekt der Koordination des Gehens auf das nachfolgende Erleben und kooperative Verhalten (nach Daten aus 16, S. 2).

sangen die Teilnehmer in den Dreiergruppen das per Kopfhörer in verschiedenen Geschwindigkeiten eingespielte Lied und bewegten den Becher entsprechend auch asynchron dazu. Den Teilnehmern in allen Gruppen (d. h. unter allen vier Bedingungen) wurde vorher gesagt, dass sie möglicherweise verschiedene Versionen des Liedes „O Kanada“ hören würden, was faktisch jedoch nur in der vierten Bedingung (asynchron) der Fall war.

Wie die Ergebnisse der nach dieser Prozedur gespielten Runden des Weak-LinkSpiels zeigten, führte gemeinsames Singen mit und ohne gemeinsames, koordiniertes Bewegen zur Wahl höherer Zahlen, also zu einem größeren Vertrauen in die jeweils anderen beiden Mitspieler in der Dreiergruppe. Beides führte also zu mehr Koope- ration im Vergleich zu asynchronem Singen und Bewegen oder zum einfachen Stillsitzen (weder singen noch bewegen). Das gemeinsame Bewegen erwies sich damit als überflüssig, denn das gemeinsame Singen allein sorgte bereits für mehr Kooperation. Es geht bei der Koordination also nicht unbedingt um grobmotorisches Handeln, sondern um synchrones Agieren, das nicht von synchronen Bewegungen von Rumpf, Armen oder Beinen begleitet sein muss.

In einem dritten Experiment wurde dies dann nochmals bestätigt, wobei die Effekte auf eine andere Weise, nämlich mit dem sogenannten Public Goods Game, gemessen wurden. Bei dem in Dreiergruppen durchgeführten Spiel geht es um Folgendes: Jeder bekommt 10 Plastik-Spiel-Chips, die man 
entweder für sich behalten oder in einen „gemeinsamen Topf“ geben kann. Für jeden Chip, den man behält, bekommt man 0,5 US Dollar, für jeden Chip im gemeinsamen Topf erhält jeder Spieler 0,25 US Dollar. Wenn jeder Spieler alles für sich behält, bleiben ihm insgesamt also 5 US Dollar; wenn hingegen alle alles in den Topf geben, erhält jeder 7,5 US Dollar ( 3 x 0,25 x 10). Es lohnt sich also, sich für die Gruppe einzusetzen, aber nur dann, wenn die anderen das auch tun. Allgemein formuliert geht es bei dem Public-Goods Game darum, eigene Mittel zu investieren, damit es der Gemeinschaft besser geht. Wer nicht investiert, profitiert dennoch von den Investitionen der anderen, was als Problem „Trittbrettfahren“ (engl: free rider problem) bekannt ist. Dieses gibt es in jeder Gemeinschaft, weswegen sehr viele Studien dazu gemacht wurden, unter welchen Bedingungen sich Menschen in diesem Spiel wie verhalten.

Wer nicht investiert, profitiert dennoch von den Investitionen der anderen.

Die Ergebnisse des über 5 Runden von 35 Dreiergruppen $(\mathrm{n}=105$, mittleres Alter 21 Jahre, 60\% weiblich) durchgeführten Spiels sind in Abbildung 4 dargestellt. Wie man sieht, ist das Ausmaß der Kooperation in den Gruppen, die zuvor gemeinsam sangen - also sowohl nach synchronem Singen als auch nach synchronem Singen und Agieren) (Plastikbecher im Rhythmus bewegen) - größer als nach der Kontrollbedingung (weder singen noch Bewegung) oder nach der asynchronen Bedingung.

Bei den Teilnehmern in den Gruppen mit asynchroner Bedingung und Kontrollbedingung kam es beim Vergleich der in den gemeinsamen Topf gegebenen SpielChips zwischen Runde 1 und Runde $5 \mathrm{zu}$ einer signifikanten Abnahme. Diese war in den synchronen Gruppen nicht beobachtbar. Diese Persistenz des prosozialen Verhaltens ist insofern bemerkenswert, da man aus früheren Studien bereits wusste, dass der in diesem Spiel in den gemeinsamen Topf gegebene Anteil über die gespielten Runden hinweg sinkt. Ein Grund hierfür könnte sein, dass unkooperative Mitspieler die Motivation zur Kooperation der anderen Spieler mindern. Nach vorherigem Singen geschieht dies nicht, was für eine gewisse Nachhaltigkeit der Effekte spricht.

Die Teilnehmer in den beiden synchronen Gruppen berichteten in einem nach dem Spiel bei allen Teilnehmern durchgeführten Fragebogen ein signifikant stärker ausgeprägtes Gefühl, im gleichen Team zu sein. Für die Stärke dieses Wir-Gefühls ließ sich in den Runden 3 bis 5 statistisch ein moderierender Effekt nachweisen, der sich auf die Menge der in den gemeinsamen Topf gegebenen Spiele-Chips auswirkte. Glücksgefühle (auch mittels Fragebogen erfasst) hatten hingegen keinen solchen Effekt, d. h. der Ausgang des Experiments war nicht einfach dadurch zu erklären, dass die Teilnehmer besser gestimmt waren.

Die Autoren kommentieren dies wie folgt: „Unsere Ergebnisse zeigen, dass kulturelle Praktiken, die synchrone Aktionen beinhalten (wie Musik, Tanz oder Marschieren) einer Gruppe dabei helfen, das Problem der Trittbrettfahrer [Menschen, die nichts zur Gruppe beitragen aber von ihr profitieren] zu mindern und durch Koordination ihren möglicherweise aufwändigen sozialen Aktivitäten zum Erfolg zu verhelfen. Rituale synchroner Aktionen haben wahrscheinlich manchen kulturellen Gruppen einen Vorteil ihrer gesellschaftlichen Evolution ermöglicht, was dazu geführt hat, dass diese überlebten, wo andere versagt haben“ (16, S. 5). ${ }^{3}$

Dass gemeinsames Tanzen prosoziale Auswirkungen hat, konnten Diane Ehlers und ihre Kollegen im Rahmen eines Experiments mit 69 Erwachsenen nachweisen. Das Ausmaß von Stress und Einsamkeit nahm zugleich signifikant ab (3). Eine weitere Studie konnte zeigen, dass es nicht auf die tatsächlich erreichte Synchronizität der Bewegungen ankommt, sondern dass es letztlich die Intention $\mathrm{zu}$ koordiniertem Handeln ist, die ausreicht, um für mehr Kooperation $\mathrm{zu}$ sorgen. Anders ausgedrückt: Auch passionierte „Nichttänzer" können durch Tanzen (oder Tanzversuche) für mehr Kooperation sorgen!
Es kommt nicht auf die erreichte Synchronizität der Bewegungen an, sondern es ist die Intention zu koordiniertem Handeln, die ausreicht, um für mehr Kooperation zu sorgen.

Mittlerweile liegt eine ganze Reihe von Studien zu den Auswirkungen von synchronem Handeln vor $(2,8,10,14)$ Eine Metaanalyse von 60 Experimenten ergab einen Effekt von $49 \%$ mehr prosozialer Einstellung und $45 \%$ mehr prosozialem Handeln (12).

Schon bei vierjährigen Kindern lässt sich der prosoziale Effekt gemeinsamen Musizierens oder Singens nachweisen, wie die Arbeitsgruppe um Michael Tomasello vom Institut für Evolutionäre Anthropologie in Leipzig bereits ein Jahr nach der gerade eben beschriebenen Untersuchung zeigen konnte (7). Mithilfe einer standardisierten komplexen sozialen Situation wurden spontane Hilfeleistung und spontanes kooperatives Problemlösen bei 96 Kindern im Alter von viereinhalb Jahren untersucht, nachdem sie zuvor in kleinen Gruppen (immer derselbe männliche Versuchsleiter und jeweils zwei Kinder gleichen Geschlechts aus dem gleichen Kindergarten, die sich schon kannten) gesungen und mit einem Perkussionsinstrument musiziert sowie sich dazu im Rhythmus bewegt hatten. Das prosoziale Verhalten wurde jeweils ohne Versuchsleiter bei den beiden Kindern untersucht, wobei die Situation so war, dass einer dem Anderen helfen musste bzw. man gemeinsam ein Problem lösen musste. Eine Kontrollgruppe führte $\mathrm{zu}$ nächst mit dem Versuchsleiter ähnliche Handlungen und Bewegungen aus, ohne jedoch $\mathrm{zu}$ musizieren, $\mathrm{zu}$ singen und $\mathrm{zu}$ tanzen. Danach wurden die gleichen Standardsituationen (spontanes Helfen, kooperatives Problemlösen) durchgespielt.

Gemeinsames Musizieren fördert signifikant mehr prosoziales Verhalten, sowohl im Hinblick auf spontane Hilfeleistungen als auch auf kooperatives Problemlösen.

Wie die Auswertung der Daten zeigte, bewirkte das gemeinsame Musizieren signifikant mehr prosoziales Verhalten, sowohl 


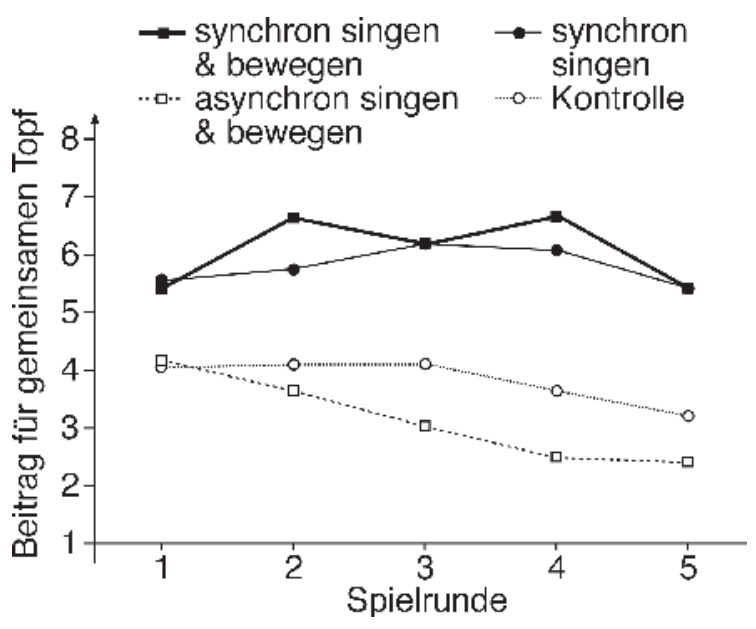

Abb. 4 Ergebnis des dritten von Wiltermuth und Heath durchgeführten Experiments. Für die 5 gespielten Runden getrennt wurde die mittlere Anzahl der Spiel-Chips, die in den gemeinsamen Topf gegeben wurden, getrennt nach den vier Bedingungen, aufgetragen. Ab Runde 2 sind die Unterschiede zwischen den oberen beiden und den unteren beiden Kurven signifikant (nach Daten aus 16, S. 4).

im Hinblick auf spontane Hilfeleistungen $(\mathrm{p}<0,01)$ als auch im Hinblick auf kooperatives Problemlösen $(\mathrm{p}<0,01)$. Dabei waren die Mädchen deutlich hilfsbereiter (mit $\mathrm{p}=0,03$ signifikant) als auch kooperativer (mit $\mathrm{p}=0,01$ signifikant) als die Jungen ( $>$ Abb. 5).

Zudem zeigten sich weitere interessante Unterschiede im Verhalten der Kinder nach dem gemeinsamen Musizieren: Von denen, die nicht halfen, äußerten $70 \%$ "Ausreden“, warum das gerade jetzt nicht gehe, wohingegen dies nur bei $33 \%$ der (nicht musizierenden) Kontrollgruppe der Fall war. Beim kooperativen Problemlösen redeten die Kinder spontan miteinander (,jetzt bis du dran“, „wir haben es geschafft"), was bei den Kindern, die nicht kooperierten nicht der Fall war. Entsprechend gab es bei den Kindern, die zuvor gemeinsam musiziert hatten, eine höhere Anzahl kommunikativer Äußerungen (Mittelwert: 6,4) als bei den Kindern der

3 „Our results suggest that cultural practices involving synchrony (e.g., music, dance, and marching) may enable groups to mitigate the free-rider problem and more successfully coordinate in taking potentially costly social action. Synchrony rituals may have therefore endowed some cultural groups with an advantage in societal evolution, leading some groups to survive where others have failed."
Kontrollbedingung (Mittelwert: 3,4; der Unterschied war mit $\mathrm{p}=0,02$ statistisch signifikant).

Angesichts der in dieser kleinen Übersicht beschriebenen prosozialen Auswirkungen von gemeinsamem Musizieren, Singen und Tanzen stimmt unser derzeitiger gesellschaftlicher Umgang mit Musik nachdenklich: Der Unterricht von Musik hatte noch nie einen besonders hohen Stellenwert, wird aber sowohl systematisch (immer weniger Stunden Musikunterricht in den Lehrplänen) als auch pragmatisch (immer mehr Musikunterricht wird von fachfremden Lehrern unterrichtet bzw. fällt an Schulen aus) eher weiter reduziert als gefördert.

Musik wird immer seltener aktiv produziert und immer häufiger passiv konsumiert. Der passive Konsum von Musik erfolgt überwiegend individuell per Kopfhörer. Dies konterkariert die prosozialen Auswirkungen von gemeinsamen und aktivem Musizieren.

Ebenso wie wir das Rad nicht neu erfinden brauchen, müssen wir Musik nicht erfinden. Es gibt sie schon - seit Jahrzehntausenden! Aber in einer Zeit abnehmender Empathie und Gemeinschaftlichkeit, zunehmender Ich-Bezogenheit, sozialer Isolation und damit einhergehender Vereinsamung müssen wir uns auf ihre Bedeutung

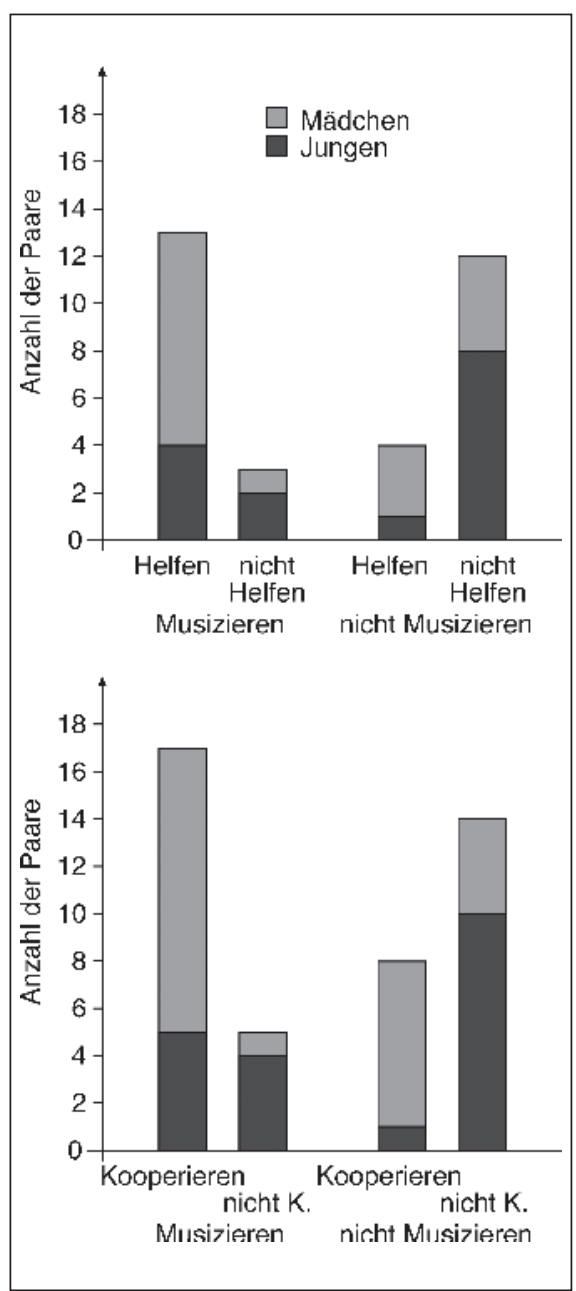

Abb. 5 Spontanes Helfen (oben) und kooperatives Problemlösen bei Vierjährigen nach vorherigem gemeinsamen Musizieren (linke Säulen) im Vergleich zu einer Kontrollgruppe (rechte Säulen), die zuvor ähnliche Aktivitäten ausführte, ohne zu musizieren (nach Daten aus 7, Figs. 2 und 3). Da es zwischen "Helfen und nicht Helfen sowie zwischen „Kooperieren und nicht Kooperieren" noch weitere Kategorien beim Outcome gab (z. B. „Abwarten" oder "ein bisschen von beidem") ergibt die Summe der Säulen nicht 48 (Anzahl der Kinder-Paare). Man sieht deutlich, dass gemeinsames Musizieren prosoziales Verhalten bahnt.

neu besinnen. Musik mögen viele Menschen als "nette Nebensache" erleben oder gar abtun. Sie ist es jedoch nicht. Die hier diskutierten Studien zeigen vielmehr ihre Bedeutung für das Funktionieren menschlicher Gemeinschaft sehr klar auf. Handeln wir danach - vor allem wenn es darum geht, wie unsere Kinder ihre freie Zeit sinnvoll gestalten sollten. 


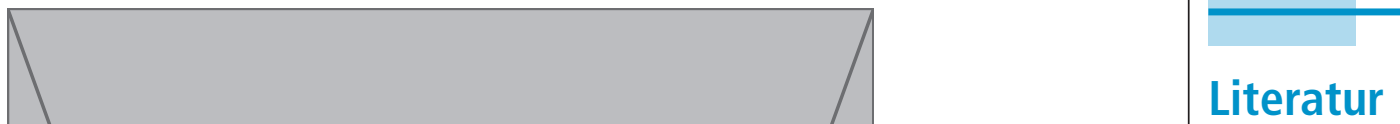

1. Anshel A, Kipper DA. The influence of group singing on trust and cooperation. Journal of Music Therapy 1988; 3: 145-155.

2. Cross L, Wilson AD and Golonka S. How Moving together brings us together: When coordinated rhythmic movement affects cooperation. Front Psychol 2016; 7: 1983.

3. Ehlers DK, Daugherty AM, Burzynska AZ, Fanning J, Awick EA, Chaddock-Heyman L, Kramer AF, McAuley E. Regional brain volumes moderate, but do not mediate, the effects of group-based exercise training on reductions in loneliness in older adults. Front Aging Neurosci 2017; 9: 110.

4. Foubert K, Collins T, De Backer J. Impaired maintenance of Interpersonal Synchronization in Musical improvisations of patients with borderline personality disorder. Front Psychol 2017; 8: 537.

5. Garland EC, Rendell L, Lamoni L, Poole MM, Noad MJ. Song hybridization events during revolutionary song change provide insights into cultural transmission in humpback whales. PNAS 2017; Jul 24. pii: 201621072.

6. Gebauer L, Witek MA, Hansen NC, Thomas J, Konvalinka I, Vuust P. Oxytocin improves synchronisation in leader-follower interaction. Sci Rep 2016; 6: 38416.

7. Kirschner S, Tomasello M. Joint music making promotes prosocial behavior in 4-year-old children. Evolution and Human Behavior 2010; 31: 354-364.

8. Kniffin KM, Yan J, Wansink B, Schulze WD. The sound of cooperation: Musical influences on cooperative behavior. J Organ Behav 2017; 38: 372-390.

9. Pearce E, Launay J, Machin A, Dunbar RI. Is group singing special? Health, well-being and social bonds in community-based adult education classes. J Community Appl Soc Psychol 2016; 26: 518-533.

10. Pearce E, Launay J, van Duijn M, Rotkirch A, David-Barrett T, Dunbar RI. Singing together or apart: The effect of competitive and cooperative singing on social bonding within and between sub-groups of a university Fraternity. Psychol Music 2016; 44:1255-1273.

11. Ravignani A, Honing H, Kotz SA. The evolution of rhythm cognition: Timing in music and speech. Front Hum Neurosci 2017; 11: 303.

12. Rennung M, Göritz AS. Prosocial consequences of interpersonal synchrony: A meta-analysis. Z Psychol 2016; 224: 168-189.

13. Rutz C, Klump BC, Komarczyk L, Leighton R, Kramer J, Wischnewski S, Sugasawa S, Morrissey MB, James R, St Clair JJ, Switzer RA, Masuda BM. Discovery of species-wide tool use in the Hawaiian crow. Nature 2016; 537: 403-407.

14. Vicary S, Sperling M, von Zimmermann J, Richardson DC, Orgs G. Joint action aesthetics. PLoS One 2017; 12: e0180101.

15. Weber RA, Camerer CF, Knez M. Timing and virtual observability in ultimatum bargaining and „weak link“ coordination games. Experimental Economics 2004; 7: 25-48.

16. Wiltermuth SS, Heath C. Synchrony and cooperation. Psychol Sci 2009; 20: 1-5. 